\title{
Environmental Affordances as a Way to Help in the Design of Videogame Worlds
}

\author{
Elisângela Vilar, Francisco Rebelo, Paulo Noriega, and Luís Teixeira \\ Ergonomics Laboratory - FMH - Technical University of Lisbon, Estrada da Costa, \\ 1499-002, Cruz Quebrada, Dafundo, Portugal \\ \{elivilar,frebelo,pnoriega, lmteixeira\}@fmh.utl.pt
}

\begin{abstract}
Videogame worlds can be read like built environments, so the approaches used to plan real environments may help the design of virtual worlds. In this way, this paper presents a pilot study that the main objective is to investigate affordances of the environment that can influence people's path selection, namely the corridor width. The main hypothesis is that the corridor width will influence people's preference regarding the path they choose in order to escape from a building (in an emergency situation). Stereoscopic images projected in a screen were presented using a constant stimulus method combined with a two-forced choice method to collect user's responses. Findings suggests that there is a tendency to bear right when users are in an "T" intersection where the right and left corridors are equal, and they tend to turn to the larger corridor regardless its direction.
\end{abstract}

Keywords: Virtual Reality, right/left bias, corridor width, affordances, videogame design.

\section{Introduction}

The orientation and navigation processes in Virtual Environments (VE) can represent an important aspect for the design of videogame worlds. They also can represent a type of environmental challenge that occurs in many games which require the player to negotiate and remember spatial configurations [1]. All VEs that simulate a complex space (which cannot be viewed from a single vantage point) will have navigation problems, and these problems may be navigators wandering aimlessly when attempting to find a place for the first time or having difficulty relocating places recently visited $[2,3]$.

Nowadays, many videogames use indoor environments where players travel through corridors, entering rooms and escaping from dangerous situations to accomplish missions. Thus, the design of these virtual buildings has increased importance for the interaction quality and for the users' performance and satisfaction.

However, the more complex a building is, the lower is the developers' knowledge about the people's navigational behavior, since the higher the complexity, the more probable it is that the designer's intuition will fail [4].

If players do not understand where they are and how to get where they need to go, they will get frustrated and instead of moving around the virtual world enjoying the 
game experience, they will look for shortcuts that make more sense for them. Therefore, a game that is not intuitive and easy to use will simply not become popular [5].

Many aspects of videogame worlds (even landscape) can be read as a built environment $[6,7]$. So, an approach to study the people's natural movement can be based on the affordances that the environment furnishes or affords the observer [8]. The conscious use of this concept in the design of environments is based on the definition of a set of affordances that express the different priorities and capabilities of a variety of users under various conditions, creating a congruence between what players realize they can do and the activities that they can perform. The formulation of this set of knowledge might be defined studying the people's interaction behavior with indoor environments, mainly with regard to the decisions taken on certain situations.

In this way, this pilot study main objective is to investigate affordances of virtual indoor corridors, namely the corridor width, as well as the right/left bias that may influence people's decision during the interaction with this environment in an emergency situation (escape from a building).

\section{Methodology}

For this pilot study, in order to investigate the corridor width and the right/left bias which can influence the users' path selection, images of virtual indoor corridors were projected using a stereoscopic projector and 3D glasses. The image sequence was presented using a constant stimulus method combined with a two-forced choice method to collect the users' responses.

The architectural issue considered was the circulation in escape routes, particularly the corridors, which the main affordance is to furnish the passage and the communication among different areas of a building.

\subsection{Design of the Experiment}

The experiment used a within-subject design and, in order to verify the influence of the independent variable (corridor width) over the people's path selection in an emergency situation, nine different corridors were designed (Fig. 1).

All corridors are composed by two corridors with a " $\mathrm{T}$ " intersection type, resulting in two opposite directional choices (left and right).

The left and the right corridors started from $2.00 \mathrm{~m}$ of width; the alternative corridors (left/right) had their width increased in $50 \mathrm{~cm}$ until reaching $4.00 \mathrm{~m}$ of width each.

In this way, the experimental conditions for the independent variable were: one neutral corridor with the same width for left and right corridors $(2.00 \mathrm{~m})$ and four corridors varying from $2.50 \mathrm{~m}$ to $4.00 \mathrm{~m}$, with increments of $50 \mathrm{~cm}$ for both, left and right corridors, alternatively.

Thus, nine experimental conditions were defined. The neutral corridor condition was repeated in order to guarantee the same number of trials for all experimental conditions, resulting in a total of ten stimuli. The stimuli were organized according to 
the method of constant stimuli and a forced choice between two alternatives method was used to collect answers.

All stimuli were repeated eight times, resulting in eighty trials. These trials were organized in two sequences, a pseudo-random sequence (Sequence 1) and the same sequence in an inverse order (Sequence 2). Thus, two blocks of sequences with forty trials each were presented for all participants, in an alternated order.

The stimulus was a static image of a corridor and had $1400 \mathrm{~ms}$ of duration with an inter-stimulus interval varying from 800 to $1000 \mathrm{~ms}$ (it was presented to the participants as a gray screen between each corridor image).

The stimulus disappeared as soon as participants push the gamepad button or when the stimulus time was finished. After the end of each stimulus there was still an extra $800 \mathrm{~ms}$ time to collet participant's choice.

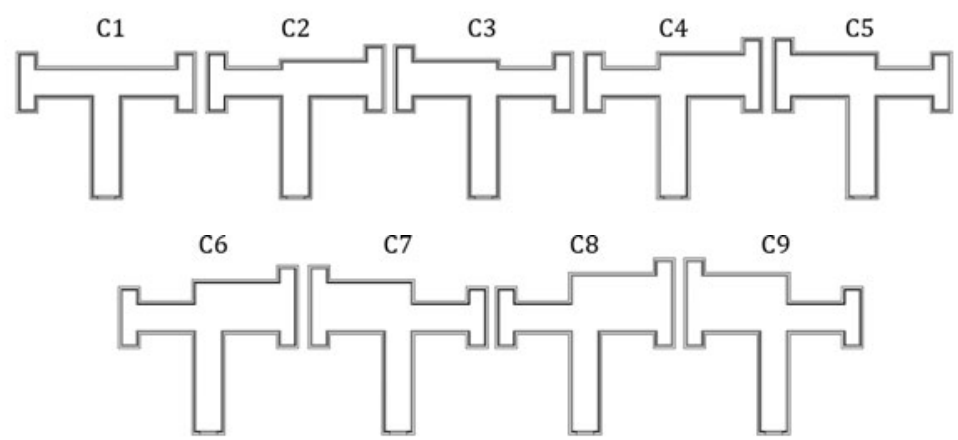

Fig. 1. Nine experimental conditions for corridor width in " $T$ " intersection shape

\subsection{Virtual Environments}

For the experiment, images of virtual corridors were projected in a screen. Thus, VE's were developed in a way that allows simulating the proposed experimental conditions.

The main requirements to the design of the corridors were:

- Existence of a point for the decision-taking related to the route to follow with two alternative paths (left $\mathrm{x}$ right) - Use of "T" intersection types corridors;

- Maintaining the decision point - same distance from the beginning to the decision point for all conditions;

- Constant width for the main corridor and for one of the two alternative paths;

- Variable width in the other alternative path (adding $50 \mathrm{~cm}$ as width variation);

- Uniform light for all corridor width - Do not use effects of light and shadow;

- Avoid environmental cues - no texturized walls or floor, use of solid colors;

- Insert elements to increase depth perspective - ceiling moldings, wainscoting, baseboards;

- Use of symmetrical elements. 
Firstly, 2D plans, which were the base structure of the VE's, were designed using software AutoCad $2008^{\circledR}$. These 2D plans were exported to 3D Studio Max ${ }^{\circledR}$ in order to model the $3 \mathrm{D}$ environments ( 9 corridors). In this phase some elements - such as colors on walls and floor, ceiling moldings, wainscoting and baseboards - were inserted in the corridors to increase their realism (Fig. 2). The modeled environments were then exported using plug-in called OgreMax 2.1.2, to be used by the ErgoVR software [9].
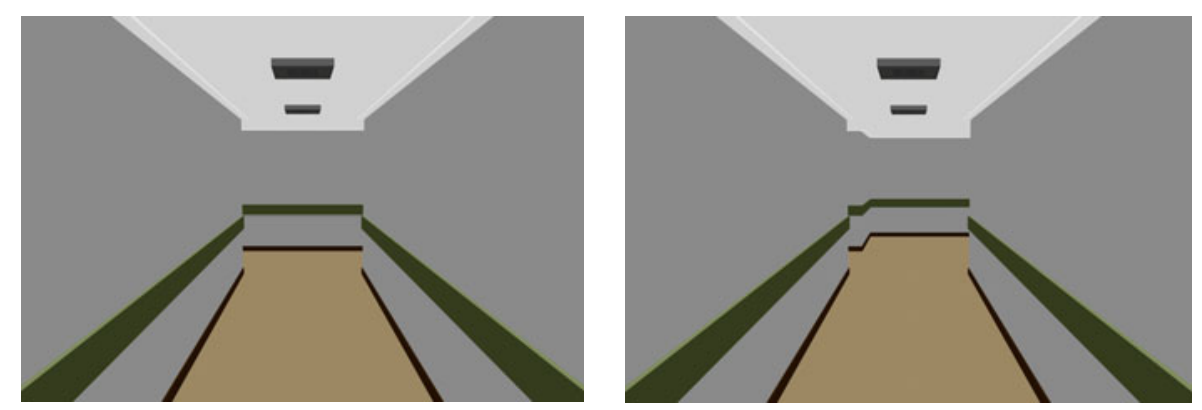

Fig. 2. Neutral corridor condition and $4 \mathrm{~m}$ width right corridor condition as they were presented by ErgoVR

\subsection{Experimental Settings}

The Virtual Reality (VR) system used for the experimental tests was comprised by the Lightspeed DepthQ 3D video projector and pair of MacNaughton Inc's APG6000 shutter glasses.

The answers were collected using the Thrustmaster FireStorm Dual Analogue 3 Gamepad, where the functional buttons at right were used to select the answers regarding to the chosen direction (left and right).

The projected images' size was $1.72 \mathrm{~m}$ (horizontal) $\times 0.95 \mathrm{~m}$ (vertical) and the observation distance (distance between the observer and the projected image) was $1.50 \mathrm{~m}$ resulting in a $35.2^{\circ}$ of vertical Field-of-view (FOV) and $59.6^{\circ}$ of horizontal FOV.

All participants remained standing during the experimental session at the same position (marked on the floor) to ensure the same observation distance. The experimenter visually monitored participants and took notes.

\subsection{Sample}

Eleven subjects, one male and ten females, aged between 20 and 68 years old (mean age $=36 ; \mathrm{SD}=14.5)$ participated in this pilot study. Ten participants $(91 \%)$ declared through a questionnaire the use of the right hand as dominant hand and one $(9 \%)$ declared the use of the left hand as dominant. All of them were graduated or undergraduated. Half of the participants was firstly assigned to choose the direction in Sequence 1 followed by the sequence 2 and the other half did the inverse order. 
The total sample observations comprise of 880 answers that the participants had to choose during Sequence 1 and Sequence 2.

\subsection{Procedure}

The experimental session was made with each participant separately. Firstly, the participant was asked to sign an Informed Consent Form and advised he/she could stop the experimental session at any time he/she wanted to. The mean duration of each experimental session was 20 minutes. Participants were unaware of the real objective of the experiment. They were told that they should choose one alternative of available paths as fast as possible, as they were in an emergency situation.

The experimental session begun with a brief training session, where the participant had some explanation about the experiment and saw the type of decision he/she should have to take. In this training session participants were told that they must choose between two alternatives of paths, i.e. the one which represents the one they would take if they were escaping from a building in an emergency situation.

The training session also comprised of two practical tests with a sequence of images like those of the experimental test. In the first practical test, the participant was asked to show with his/her hands the both alternatives of corridors he/she was seeing in the image. This was done in order to ensure that the participants realized the alternative of paths that they had in front of them. The second practical test was made in order to make participants familiar with: i) the gamepad buttons which they used to choose their direction and, ii) the time available for their answer since the time used for the training session was the same used for the experimental tests.

Experimental tests began after participants gave their answers in the available time and declared they were comfortable with the gamepad buttons.

For the experimental test, the participant was assigned to the first sequence of 40 trials. Finishing the first sequence and after a 5minutes break, the participant was assigned in to the second sequence.

At the end of the experimental session a demographic questionnaire was applied to collect information such as age, gender, occupation and dominant hand. Participants were also asked to answer questions related to the experimental test.

\section{Results and Discussion}

The participants' choices regarding the direction they would take for each presented condition considering they were escaping from a building in an emergency situation are presented in Table 1. The results represent the participants' direction choice for all trials (880) and were analyzed globally. There were considered as invalid all missing or impossible answers (when participant pushed a wrong gamepad directional button). Some studies suggest that in ordinary condition (relatively symmetrical buildings) there is a tendency for the public to bear to the right $[10,11]$. Robinson [10] stated that this trend (about $75 \%$ of the visitors bear to the right and about $25 \%$ to the left) had been observed in different museums across the United States of America. Scharine and McBeath [11] found that $61.6 \%$ of people who had to walk along a corridor formed by a set of paralels bookshelves turned right at the end of the corridor and 
$38.4 \%$ went to the left. Condition $\mathrm{C} 1$ represents the neutral condition, with the same width $(2.00 \mathrm{~m})$ for all corridors. For the studied situation, results suggest that there is a tendency for the people to prefer the right $(64.1 \%)$ instead turning to the left $(35.9 \%)$.

Table 1. Participants' choice for experimental condition regarding the direction

\begin{tabular}{cccccccc}
\hline Conditions & Invalid & Left & Right & $\begin{array}{c}\text { Total without } \\
\text { Invalids }\end{array}$ & $\begin{array}{c}\% \\
\text { Invalids }\end{array}$ & $\begin{array}{c}\% \\
\text { Left }\end{array}$ & \% Right \\
\hline C1 & 6 & 61 & 109 & 170 & 3,4 & 35,9 & 64,1 \\
C2 & 1 & 11 & 76 & 87 & 1,1 & 12,6 & 87,4 \\
C3 & 1 & 60 & 27 & 87 & 1,1 & 67 & 31 \\
C4 & 0 & 10 & 78 & 88 & 0 & 11,2 & 88,6 \\
C5 & 0 & 65 & 23 & 88 & 0 & 74 & 26,1 \\
C6 & 0 & 11 & 77 & 88 & 0 & 12,5 & 87,5 \\
C7 & 1 & 79 & 8 & 87 & 1,1 & 90,8 & 9,2 \\
C8 & 1 & 14 & 73 & 87 & 1,1 & 16,1 & 83,9 \\
C9 & 0 & 69 & 19 & 88 & 0 & 78,4 & 21,6 \\
\hline
\end{tabular}

The graphic at Fig. 3 shows the percentage of choices favoring the corridor at the right side. Axis $x$ represents the amount of increment added to the width of the corridors. Negative values indicate that these increments were made on the corridor at the left side. The value " 0 " represents the corridor with no width difference between the corridors on the left or the right sides (neutral condition C1). Axis $y$ represents the percentage of choices favoring the corridor on the right side regardless they were the wider or not. Considering the value 0 (C1), we can notice that the $64.1 \%$ of the choices were to the corridor on the right side.

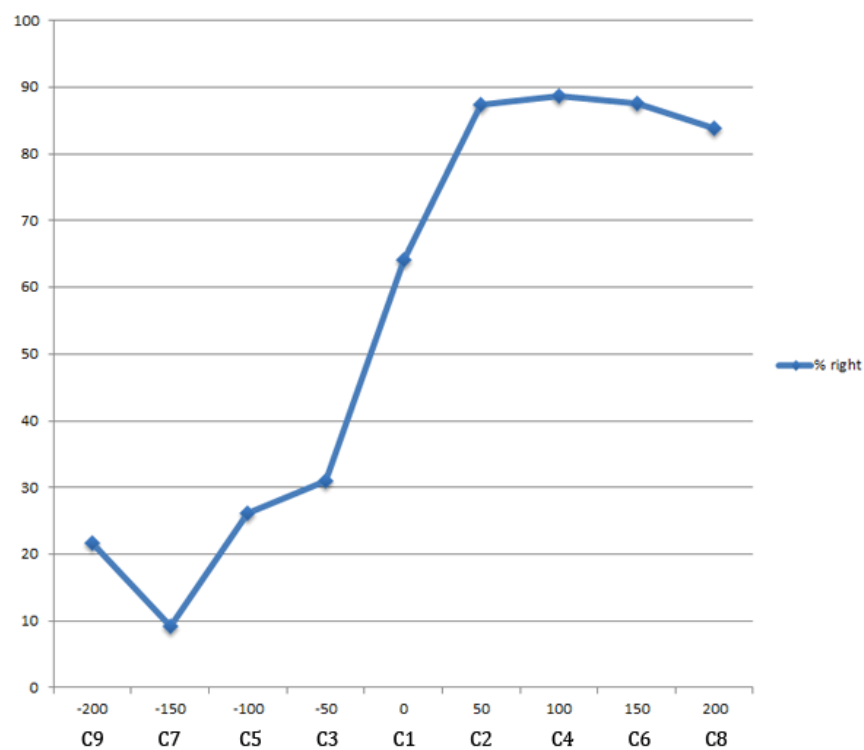

Fig. 3. Graphic with the percentage of choices favoring the corridors at the right side 
These findings are in line with previous studies, allowing supporting the hypothesis that people tend to turn right. These findings also contribute to validate the methodology and the setup used in this pilot study to investigate issues considering the variables related to the user's choice regarding the direction.

Fig. 3 also shows that the right bias tends to decrease when the corridor has an increment over its width. The wider the corridor, the lower the tendency to turn right regardless the width. This tendency is contradicted for the largest corridor of the experimental conditions. Curiously, some participants after the experimental session informally reported that there were corridors which were so large that they did not chose to go through them because they will feel like they were alone in that large and empty space. In this way, although the design of this study does not allow verifying this hypothesis, in some way, large empty corridors might provoke bad feelings decreasing their use by visitors. However, despite the plausibility of this hypothesis, the number of participants in this pilot study is small and it may have affected the results.

Considering the corridor width, when the corridor has an increment of $50 \mathrm{~cm}$ in width, the effect of this variable is noticed. Condition C3 (Table 1) represents the case where the left corridor had $2.50 \mathrm{~m}$ of width while the right corridor continued with $2.00 \mathrm{~m}$ of width. In this case, $67 \%$ of participants chose to bear left. The effect of corridor width increases until the increment of $150 \mathrm{~cm}(\mathrm{C} 6 / \mathrm{C} 7)$, as can be seen on Fig. 4.

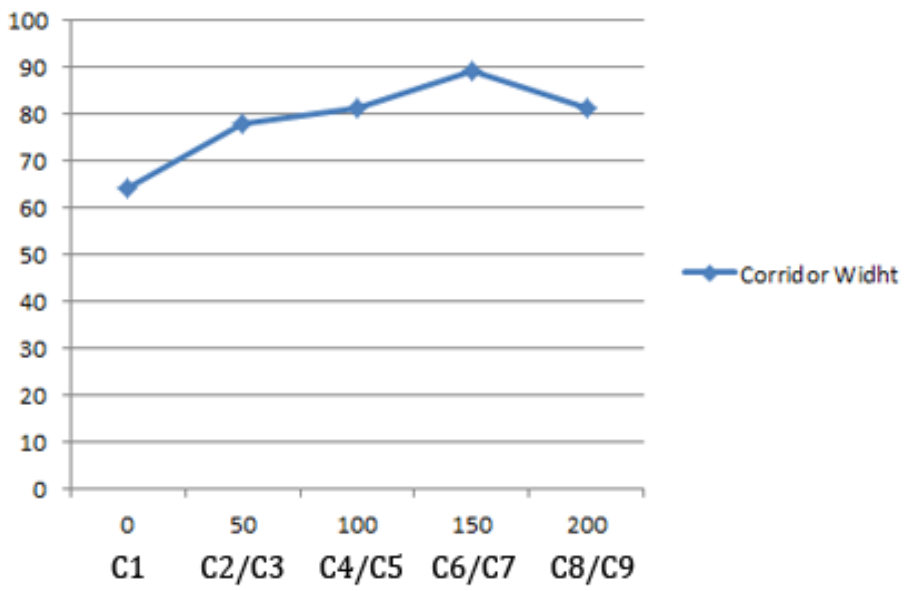

Fig. 4. Graphic with the percentage of choices favoring the large corridors for both sides together

\section{Conclusion}

A corridor affords passage and communication from one area to another; however when videogame players are confronted with a situation where they have to choose 
between two options of corridors, some environmental cues can act as attractiveness factor. In this way, the main objective of this pilot study is to verify the hypotheses that people prefer to bear to right and to wider corridors.

Early studies carried out using the real world as interaction environment concluded that in relatively symmetrical buildings or corridor situations, there is a tendency for users to bear to the right $[10,11]$. The findings of this pilot study allow corroborating with the early studies results and also indicate that the methodology and the VR setup used in this pilot study are consistent and robust enough to study variables related to the user's choice regarding to the direction.

Regardless left/right bias, when the corridor has an increment of $50 \mathrm{~cm}$ in the width, the effect of this variable is noticed. Findings of this pilot study allow supporting the hypothesis that users in emergency situation prefer wider corridors. However, data surprisingly show that the choice favoring the wider corridor decreased in the conditions with higher increment value $(200 \mathrm{~cm})$ suggesting that until certain circumstances the increment in the width of corridor can act as an attraction factor, but if this increment is somewhat higher the result can be the opposite. Despite the plausibility of this hypothesis, the number of participants in this pilot study could be small and it may have affected the results. More research is needed in this area since no studies were found about users directional choices considering variables as width, height, length, lighting, and color.

Affordances are environmental properties which have some meaning to guide the observer's behavior [12,13], and, according to Venturi and Brown [14], the activities of people into buildings can be seen as patterns of use. In this way, the study of this patterns may be an useful help to game designers to understand people natural movements and predict their behavior, in order to create virtual environments that encompass: a non-obvious way of orientation (opposite to following e.g. arrows, that can make the game boring), an scenario where the user's path selection is undercontrol and a space that continue being a challenge to the players.

Some questions might be raised with this pilot study. Concerning the sample, this pilot study had a total of 880 observations ( 80 per participant); however increasing the number of participants and/or the number of trials they performed might produce some effects on the results. In this way, a large sample probably will ensure more robust results in future works.

Another issue to consider is that fixed images projected in a screen, as the setup used in this pilot study, may lack ecological validity. They do not reproduce the natural way of space use; consequently, they do not have high levels of immersion, representing another point that should be improved for future works. When people interacts with an indoor environment they walk and look around to find environmental cues to help with their wayfinding decisions, in this way, using more dynamic scenarios - like videos or animations - with visualization through headmounted displays and with free visual exploration of the environment will allow a higher level of ecological validity.

Data related to the presence and levels of immersion were not considered for this pilot study, however these variables are also important issues to be investigated. Posttest questionnaires are an easy and useful way to measure presence and level of immersion and their use for future work should be considered. 
The contribute of VR systems in the studies related to patterns of space use, namely the people's path selection, can be considered as an important issue since VR systems shown to be an effectiveness way to measure users' decision.

Acknowledgments. This research was supported by the Portuguese Science and Technology Foundation (FCT) grants (PTDC/PSI-PCO/100148/2008 and SFRH/BD/ 38927/2007).

\section{References}

1. McGregor, G.L.: Situations of Play: Patterns of Spatial Use in Videogames. In: Proceedings of DiGRA 2007 International Conference Situated Play, Tokyo, pp. 537-545 (2007), http: / /www.digra.org/dl/display_html?chid=07312.05363.pdf (retrieved)

2. Darken, R.P., Peterson, B.: Spatial Orientation, Wayfinding and Representation. In: Stanney, K.M. (ed.) Handbook of Virtual Environments: design, implementation, and applications, pp. 493-518. Lawrence Erlbaum Associates, New Jersey (2002)

3. Darken, R.P., Sibert, J.L.: Wayfinding strategies and behaviors in large virtual worlds. In: Proceedings of the SIGCHI 1996 conference on Human factors in computing systems, pp. 142-149. ACM, New York (1996)

4. Conroy, R.: Spatial navigation in immersive virtual environments. Unpublished Dissertation, University of London, London (2001)

5. Aarseth, E.: Allegories of Space. In: Borries, F., Walz, S.P., Böttger, M. (eds.) Space Time Play, pp. 44-47. Birkhäuser, Basel (2007)

6. Darken, R.P.: Wayfinding in large-scale virtual worlds. In: ACM SIGCHI 1995, pp. 4546. ACM, Denver (1995)

7. McGregor, G.L.: Architecture, space and gameplay in World of Warcraft and Battle for Middle Earth 2. In: Proceedings of the CyberGames International conference on Game research and development, Murdoch University, Australia (2006),

http://www.users.on.net/ georgia88/files/

Architecture, $\% 20$ Space 20 and $\% 20$ Gameplay $\% 20-$

\%20Georgia\%20Leigh\%20McGregor.paf (retrieved)

8. Gibson, J.J.: The Ecological Approach to Visual Perception. Lawrence Erlbaum Associates, Boston (1986)

9. Teixeira, L., Rebelo, F., Filgueiras, E.: Human Interaction Data Acquisition Software for Virtual Reality. In: Kaber, D., Boy, G. (eds.) Advances in Cognitive Ergonomics, pp. 793801. CRC Press, Boca Raton (2010)

10. Robinson, E.S.: The Psychology of Public Educations. American Journal of Public Health 23(2), 123-128 (1933)

11. Scharine, A.A., McBeath, M.K.: Right-Handers and Americans Favor Turning to the Right. Human Factors: The Journal of the Human Factors and Ergonomics Society 44(2), 248-256 (2002)

12. Stoffregen, T.A.: Affordances and Events. Ecological Psychology 12(1), 1-28 (2000)

13. Turvey, M.T.: Affordances and Prospective Control: An Outline of the Ontology. Ecological Psychology 4(3), 173-187 (1992)

14. Venturi, R., Brown, D.S.: Architecture as Signs and Systems: for a mannerist time. Massachusetts. Belknap Press of Harvard University Press, Cambridge (2004) 